\title{
Niclosamide acts as a new inhibitor of vasculogenic mimicry in oral cancer through upregulation of miR-124 and downregulation of STAT3
}

\author{
XIAOXU LI ${ }^{1,2^{*}}$, ZHICHENG YANG $^{4 *}$, ZEWEN HAN $^{2}$, YANHUI WEN $^{5}$, ZEYUN MA $^{3}$ and YIXIANG WANG ${ }^{1,2}$ \\ ${ }^{1}$ Central Laboratory, Departments of ${ }^{2}$ Oral and Maxillofacial Surgery, and ${ }^{3}$ VIP Service, \\ Peking University School and Hospital of Stomatology, Beijing; ${ }^{4}$ Department of Stomatology, \\ Shandong Provincial Qianfoshan Hospital, Jinan, Shangdong; ${ }^{5}$ Department of Otorhinolaryngology-Head \\ and Neck Surgery, Dongguan Third People's Hospital, Dongguan, Guangzhou, P.R. China
}

Received June 20, 2017; Accepted December 5, 2017

DOI: $10.3892 / o r .2017 .6146$

\begin{abstract}
Tumors require nutrients and oxygen for growth and metastasis. Vasculogenic mimicry (VM) has been found as a new manner of blood supply, which is characterized as the formation of tumor cell-lined vessels instead of endothelial vessels. This is why angiogenesis agents targeted to endothelial cells show a limited efficacy. Up to this point, there is no effective drug reported for inhibiting VM formation. Niclosamide is an oral anti-helminthic drug used to treat human tapeworms. Recent studies have indicated that niclosamide has broad applications for cancer and other diseases. In this study, we found that niclosamide could not only inhibit proliferation and promote apoptosis of oral cancer cells, but also inhibited $\mathrm{VM}$ formation in vitro and in vivo through downregulation of the expression of VM-related genes VEGFA, MMP2, ROCK1 and Cdc42. In addition, niclosamide upregulated miR-124 and downregulate phosphorylated (p)-STAT3 expression. Further studies showed that, the stable highly expressing miR-124 cell line HN6-miR-124, such as niclosamide, could downregulate p-STAT3 expression. Moreover, HN6-miR-124 showed lower mobility, invasiveness and VM formation ability than control cells. Taken together, our study suggests that niclosamide functions as a new inhibitor of VM in oral cancer through
\end{abstract}

Correspondence to: Dr Yixiang Wang, Central Laboratory, Peking University School and Hospital of Stomatology, 22 Zhongguancun South Avenue, Haidian, Beijing 100081, P.R. China

E-mail: kqwangyx@bjmu.edu.cn

Dr Zeyun Ma, Department of VIP Service, Peking University School and Hospital of Stomatology, 22 Zhongguancun South Avenue, Haidian, Beijing 100081, P.R. China

E-mail:mzy101@sina.cn

*Contributed equally

Key words: oral cancer, niclosamide, vasculogenic mimicry, miR-124, STAT3 upregulation of miR-124 and downregulation of STAT3, providing a new and safe potential drug candidate for anti-VM therapy.

\section{Introduction}

Oral cancer is the 9 th most common cancer among males and the 14th among females in the US according to a recent study (1). Approximately $90 \%$ of oral cancer cases are oral squamous cell carcinoma (OSCC) (2). As early stage OSCC is asymptomatic, patients with OSCC are often diagnosed at the middle and advanced stages resulting in a poor prognosis. Although multidisciplinary treatment strategy has been made in OSCC, the 5-year overall survival of OSCC is still less than $50 \%$. Little improvement has been made in the last 35 years (3). Thus, it is an urgent need to achieve a better understanding of the mechanisms of OSCC and discover effective therapeutic targets.

Ten hallmarks of cancer have been published in cells (4). One of these is angiogenesis. The newly-formed endothelial vessels of which are thought to arise by sprouting of preexisting capillaries, supply nutrients and oxygen for tumor growth and metastasis (4). Therefore, an astonishing outpouring of anti-angiogenic inhibitors has been developed as a promising method to 'starve' tumors in the past decade. However, anti-angiogenesis targeted to endothelium have shown limited efficacy. This indicates that there may be other supplementary blood supply patterns to nourish tumors. In 1999, Maniotis and his colleagues found a new manner of blood supply named vasculogenic mimicry (VM), which is independent of traditional angiogenesis (5). VM is challengeable and complementary to traditional angiogenesis. The structure of VM lacks endothelial lining, instead of aggressive cancer cells forming vessel-like structure in patients' aggressive tumors (5). The features of positive periodic acid-schiff (PAS) and negative CD34 are regarded as the golden standard for tumor cell-lined VM. Myriad of studies have contributed to the understanding of VM since its introduction. Collectively, VM is closely associated with metastasis and poor prognosis in many cancers including melanoma, lung cancer, hepatocellular carcinoma 
and also oral cancer (6). However, certain studies showed that traditional angiogenesis inhibitors could not inhibit the formation of VM, and even caused extracellular matrix-rich tubular network formation in vitro (7). Therefore, therapeutic strategies that target VM hold a great promise in the treatment of cancer; but up to this point, there is no effective inhibitor reported to VM.

Niclosamide is a FDA-proven oral anti-helminthic drug used worldwide against human tapeworms for approximately 50 years (8). Recently, more beneficial effects of niclosamide have been found in several diseases that are irrelevant to parasites. These diseases include cancer, type II diabetes, bacterial and viral infection, neuropathic pain, rheumatoid arthritis, and bone loss diseases (9). Detailed mechanism studies show that niclosamide exerts anticancer effect in many types of cancers including acute myelogenous leukemia, colon cancer, prostate cancer, lung cancer, breast cancer and ovarian cancer through inhibition of WNT, Notch, mTOR and STAT3 pathways (10). However, the precise mechanism underlying this anticancer activity has yet to be elucidated.

In addition, a large fraction of the genome sequences are active but only $2 \%$ of it encodes a protein according to the analysis of human genome sequence, thus the majority of transcripts are known as non-coding RNAs (ncRNAs) $(11,12)$. Currently, small non-coding RNAs such as microRNAs (miRNAs) have been studied extensively and their potential to regulate the gene expression and cell function have emerged as a tool for diagnosis of numerous cancers $(12,13)$. Previous studies show that microRNA-124 (miR-124) acts as a tumor suppressor in head and neck squamous cell carcinoma (14) and overexpression of miR-124 correlates with better breast cancer prognosis (15). Moreover, miR-124 has been reported to be associated with VM in cervical cancer cells (16). However, whether miR-124 is involved in the niclosamide's anticancer effect in oral cancer remains unclear.

In this study, we found that niclosamide inhibited proliferation and promoted apoptosis of oral cancer cells by using two OSCC cell lines WSU-HN6 and Tca83. Furthermore, niclosamide not only inhibited VM formation of OSCC cancer cells in vitro, but also decreased the tumor size and the number of VM in vivo. Molecular assays demonstrated that, niclosamide markedly downregulated VM-associated genes VEGFA, MMP2, ROCK1 and Cdc42, whereas, it upregulated miR-124 and downregulated p-STAT3 significantly. Intriguingly, like niclosamide, stably expressing miR-124 OSCC cancer cell line inhibited the p-STAT3 expression. Moreover, the stable cell line HN6-miR-124 could decrease the ability of mobility, invasiveness and VM formation. Together, our study suggests that niclosamide shows potential to be a new inhibitor of VM in oral cancer through upregulation of miR-124 and downregulation of STAT3.

\section{Materials and methods}

Cell culture. WSU-HN6 cells were cultured in Dulbecco's modified Eagle's medium (DMEM, Gibco, Carlsbad, CA, USA) and Tca83 cells in Roswell Park Memorial Institute (RPMI)-1640 medium (Gibco), supplemented with $10 \%$ fetal bovine serum (FBS; Hyclone). They were maintained at $37^{\circ} \mathrm{C}$ in a humidified incubator with $5 \% \mathrm{CO}_{2}$.
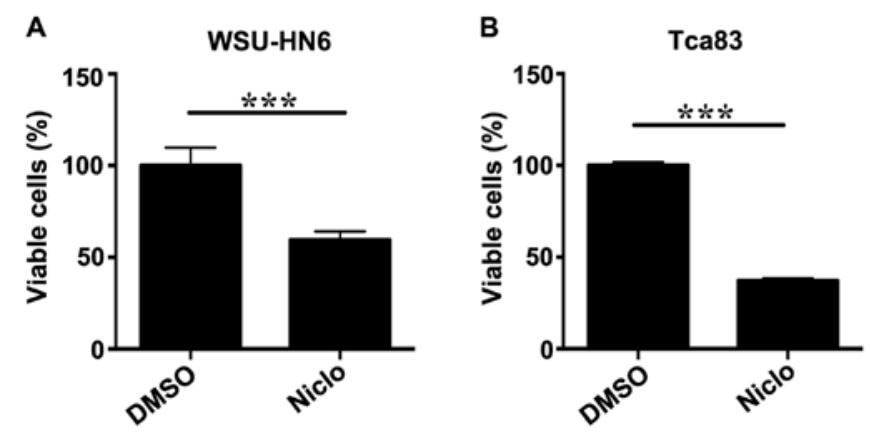

Figure 1. Niclosamide inhibits cell proliferation of oral cancer cells. Cell proliferation was detected by CCK- 8 assay. (A) Niclosamide inhibited WSU-HN6 cell line growth. (B) Niclosamide inhibited Tca83 cell line growth. Data are expressed as the means \pm SD of three independent experiments, ${ }^{* * *} \mathrm{P}<0.001$.

Cell proliferation assay. The cell proliferation was determined in vitro using Cell Counting Kit-8 (CCK-8) assay. Briefly, cells were seeded at a density of $1 \times 10^{4}$ cells per well with $100 \mu 1$ growth medium in 96-well plates. Following overnight incubation, cells were cultured in complete culture medium containing $5 \mu \mathrm{M}$ niclosamide [Sigma-Aldrich, St. Louis, MO, USA, dissolved in dimethyl sulfoxide (DMSO)]. After $24 \mathrm{~h}$ of treatment, CCK-8 reagent (10 $\mu \mathrm{l})$ was added to each well for color development. Cell viability was determined photometrically at $450 \mathrm{~nm}$ using an ELx808 absorbance microplate reader (BioTek Instruments, Winooski, VT, USA).

Apoptotic assay. Apoptosis caused by niclosamide was assessed by Cell Death Detection Kit II (Roche Diagnostics, Indianapolis, IN, USA). Briefly, WSU-HN6 and Tca83 cells were treated with DMSO and $5 \mu \mathrm{M}$ niclosamide in $100-\mathrm{mm}$ dishes for $24 \mathrm{~h}$. The cells were harvested and washed with icecold PBS twice. Apoptosis was detected by flow cytometry after the cells were stained with FITC-conjugated Annexin V and propidium iodide (PI) according to manufacturer's instructions.

Generation of stably expressing miR-124 oral cancer cell line. WSU-HN6 cells were transfected with pCDNA3.2/ V5-hsa-mir-124 plasmids carrying miR-124 overexpression cassette. pcDNA3.2/V5 hsa-mir-124 was a gift from David Bartel [Addgene plasmid \#26306 (17)]. Cells were screened by G418 for 2 weeks. The cells transfected with empty vector served as control. The single colonies were picked up for detection of miR-124 expression.

Capillary-like tube formation assay. Pre-chilled 96-well plates were coated with $50 \mu 1$ Matrigel (BD Biosciences, Franklin Lakes, NJ, USA) for $1 \mathrm{~h}$ at $37^{\circ} \mathrm{C}$. Tumor cells $\left(2 \times 10^{4}\right.$ per well) were seeded in Matrigel coated plates. Then culture plates were exposed to $5 \mu \mathrm{M}$ niclosamide for $8 \mathrm{~h}$. Tubular structures were photographed under a microscope with recording digital camera (DP72; Olympus, Tokyo, Japan), and five representative fields per well were used to evaluate the ability of tube formation.

Real-time PCR. Total RNA was isolated using the TRIzol reagent (Invitrogen, Carlsbad, CA, USA). RNA $(2 \mu \mathrm{g})$ was 
WSU-HN6

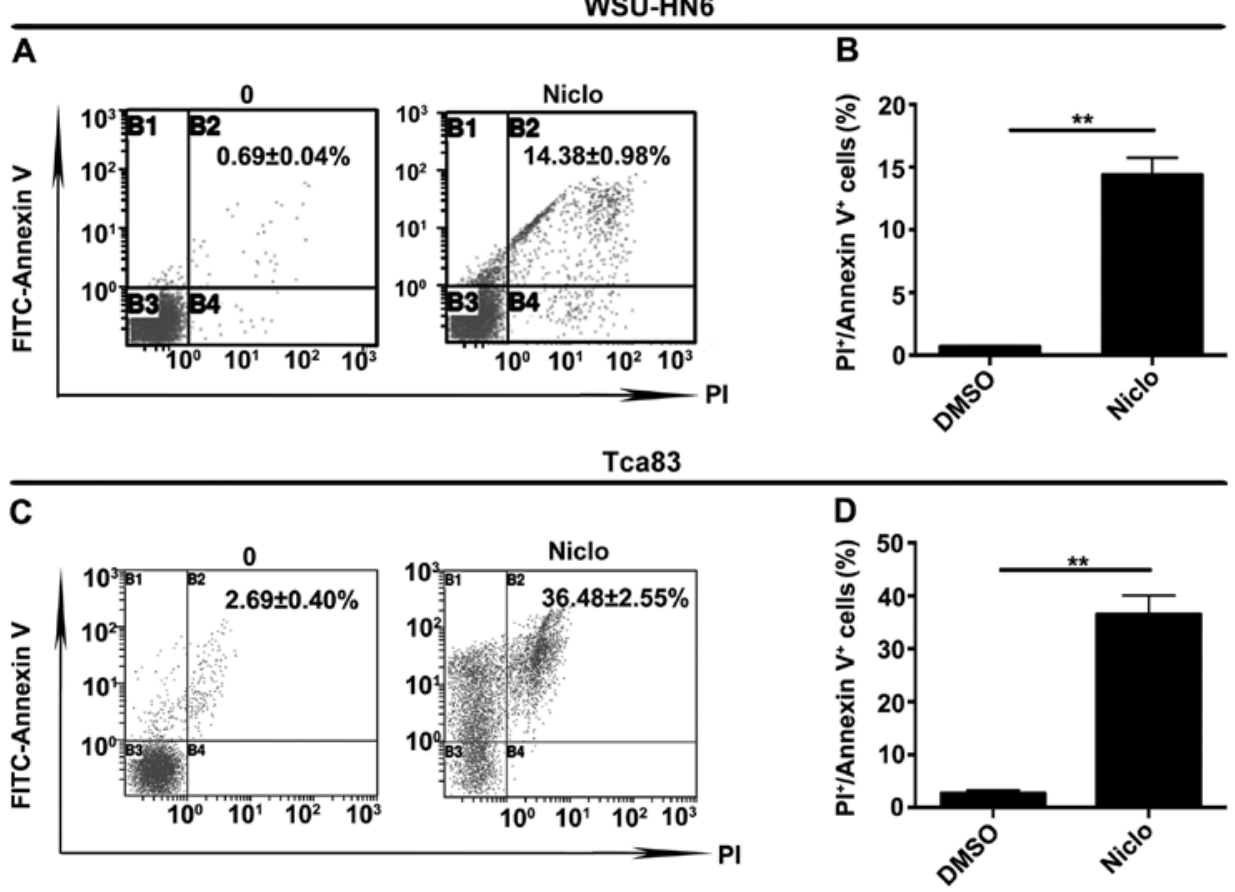

Figure 2. Niclosamide induces WSU-HN6 and Tca83 apoptosis. The level of apoptosis was evaluated using the FITC-Annexin V/PI dual-labeling technique, as determined by flow cytometry. (A and B) Niclosamide promoted apoptosis in WSU-HN6 cell line. (C and D) Niclosamide promoted apoptosis in Tca83 cell line. Data are expressed as means \pm SD from three independent experiments, ${ }^{* *} \mathrm{P}<0.01$.

reversely transcribed into cDNA with a moloney murine leukaemia virus reverse transcriptase (M-MLV RTase; Promega, Madison, WI, USA). VEGFA, MMP2, Cdc42 and ROCK1 were detected with the following respective primers: VEGFA (5'-GCAGAATCATCACGAAGTGG-3' and 5'-GCA ACGCGAGTCTGTGTTTTTG-3'); MMP2 (5'-GCCCCA GACAGGTGATCTTG-3' and 5'-GCTTGCGAGGGAAGA AGTTGT-3'); Cdc42 (5'-CCATCGGAATATGTACCGA CTG-3' and 5'-CTCAGCGGTCGTAATCTGTCA-3'); ROCK1 (5'-AACATGCTGCTGGATAAATCTGG-3' and 5'-TGT ATCACATCGTACCATGCCT-3'); GAPDH (5'-ATGGGGA AGGTGAAGGTCG-3' and 5'-GGGGTCATTGATGGCAA CAATA-3'), respectively. miRNA quantifiation was performed using miDETECT A Track ${ }^{\mathrm{TM}}$ miRNA qRT-PCR kit (RiboBio, Guangzhou, China) following the manufacturer's instruction. Real-time PCR was performed using the SYBR Green master mix (Roche Diagnostics) on an ABI 7500 instrument (Applied Biosystems, Foster, CA, USA). miR-124 and U6 primers were from Ribobio. GAPDH and U6 served as mRNA and miRNA endogenous controls, respectively. The fold-change was determined as $2^{-\Delta \Delta C t}$. All real-time PCR reactions were performed in triplicate and repeated three times.

Western blot assay. Cells were harvested and lysed in RIPA buffer (Applygen Technologies, Beijing, China) with protease inhibitors (Roche Diagnostics). Protein (30 $\mu \mathrm{g})$ was loaded. Antibodies against phosphorylated (p)-STAT3 (1:1,000), total (t)-STAT3 (1:1,000), VEGFA (1:500), MMP2 (1:500), p-Cdc42 (1:1,000), and GAPDH $(1: 1,000)$ were incubated, and then membranes were placed overnight at $4^{\circ} \mathrm{C}$. Then, relevant secondary antibodies were incubated on the membranes. Immunoreactive bands were visualized with a chemiluminescence detection system (Applygen Technologies).
Wound healing assay. Cells $\left(5 \times 10^{5}\right)$ were cultured as confluent monolayers, and then serum-starved for $30 \mathrm{~h}$ followed by wound scratching across the well with a $200-\mu 1$ pipette tip. The detached cells were removed by D-Hanks. Then the cells were treated with $5 \mu \mathrm{M}$ niclosamide for indicated times. The monolayer was photographed at a magnification of x10 using an inverted microscope (Nikon Corp., Tokyo, Japan) and the speed of the cell movement was calculated.

Transwell invasion assay. A Transwell chamber plate $(8 \mu \mathrm{m}$ pore size, Millipore, Bedford, MA, USA) with a polycarbonate membrane coated with $100 \mu 1$ of diluted Matrigel (BD Biosciences, Minneapolis, MN, USA) was used for the cell invasion assays. Briefly, $1 \times 10^{5}$ of 24 -h serum-starved cells in $100 \mu \mathrm{l}$ serum-free medium were seeded in the matrigel coated upper chamber of the Transwell, while $500 \mu \mathrm{l}$ of culture medium supplemented with $20 \%$ FBS was added to the bottom chamber. Niclosamide $(5 \mu \mathrm{M})$ was added in each upper chamber. The cells were allowed to migrate for $20 \mathrm{~h}$, and then fixed with $95 \%$ ethanol, stained with $1 \%$ crystal violet (SigmaAldrich). After wiping off the non-migrated cells in the upper chamber of the Transwell, migrated cells were counted and photographed using a light microscopy at a magnification of x20 (Olympus). Ten high-power fields of each treatment were used to calculate the relative migration speed, and the experiment was repeated three times.

Oral cancer xenografts and treatment. Medical ethics committee of the Peking University Health Center approved this study. WSU-HN6 cells $\left(3 \times 10^{6} /\right.$ mouse) were s.c. injected into 6-week-old female BALB/c nude mice, 5 mice in each group. Mice were treated with vehicle control (Kolliphor ${ }^{\circledR}$ EL) and niclosamide (20 mg/kg/day i.p.). Tumor volume was 
calculated with the formula: $\mathrm{V}=\left(\mathrm{L} \times \mathrm{W}^{2}\right) / 2(\mathrm{~L}$, length; W, width). Mice were sacrificed and tumors were harvested on the 15 th day of treatment.

Periodic acid-Schiff (PAS)-CD34 dual staining. Immunostaining was applied to perform CD34 staining. PAS staining was performed using PAS staining kit (Solarbio Co., Ltd., Beijing, China). Briefly, after DAB reaction for CD34 immunostaining, the sections were treated with $0.5 \%$ periodic acid solution for $10 \mathrm{~min}$, rinsed with distilled water for $5 \mathrm{~min}$, followed by staining in periodic acid-Schiff solution for 15-30 min. After rinsing with distilled water, sections were counterstained with hematoxylin, dehydrated, cleared and mounted.

Statistical analysis. Statistical analysis was performed with GraphPad Prism v5.0 software. All data are presented means \pm standard deviation of the mean $(\mathrm{SD}), \mathrm{P}<0.05$ was considered to indicate a statistically significant difference.

\section{Results}

Niclosamide inhibits cell proliferation of oral cancer cells. To determine whether niclosamide inhibits the growth of WSU-HN6 and Tca83 cells, cell viability was determined by CCK-8 assay, respectively. After the cells were treated with vehicle DMSO or $5 \mu \mathrm{M}$ niclosamide for $24 \mathrm{~h}$, the results showed that niclosamide significantly inhibited cell proliferation of WSU-HN6 (Fig. 1A) and Tca83 (Fig. 1B) in both oral cancer cell lines.

Niclosamide induces apoptosis of oral cancer cells. To investigate whether niclosamide inhibits oral cancer cell growth through induction of apoptosis, we performed apoptosis assay as shown in Fig. 2. Cells were treated with vehicle dimethyl sulfoxide (DMSO) or $5 \mu \mathrm{M}$ niclosamide for $24 \mathrm{~h}$, respectively. The apoptosis rates of the treated cells were $0.69 \pm 0.04 \%$ and $14.38 \pm 0.98 \%$ in WSU-HN6 $(\mathrm{P}<0.05$, Fig. 2A and B), and $2.69 \pm 0.40 \%, 36.48 \pm 2.55 \%$ in Tca83 $(\mathrm{P}<0.05$, Fig. $2 \mathrm{C}$ and $\mathrm{D})$.

Niclosamide decreases capillary-like tube formation in vitro, and inhibits tumor growth and VM in vivo. To investigate whether niclosamide plays an anticancer role through VM, we carried out capillary-like tube formation assay in vitro and VM detection by PAS-CD34 dual staining in niclosamide treated WSU-HN6 xenografts in vivo. The results showed that $5 \mu \mathrm{M}$ niclosamide completely inhibited the capacity of tube formation in the OSCC cell lines WSU-HN6 (Fig. 3A and B) and Tca83 (Fig. 3C and D) in vitro. Next, we generated oral cancer xenograft mice by subcutaneously implanting WSU-HN6 cells into nude mice. We found that compared with control group, $20 \mathrm{mg} / \mathrm{kg} /$ day niclosamide significantly decreased the tumor size (Fig. 3E) and the number of VM in vivo (Fig. $3 \mathrm{~F}$ and $\mathrm{G}$ ). We performed real-time PCR and western blotting to detect VM-related gene expression including VEGFA, MMP2, ROCK1 and Cdc42 in $5 \mu \mathrm{M}$ niclosamide-treated WSU-HN6 and Tca83 cell lines. The real-time PCR result showed that niclosamide markedly inhibited VEGFA, MMP2, ROCK1 and Cdc42 expression at mRNA level in WSU-HN6 (Fig. 4A)
A
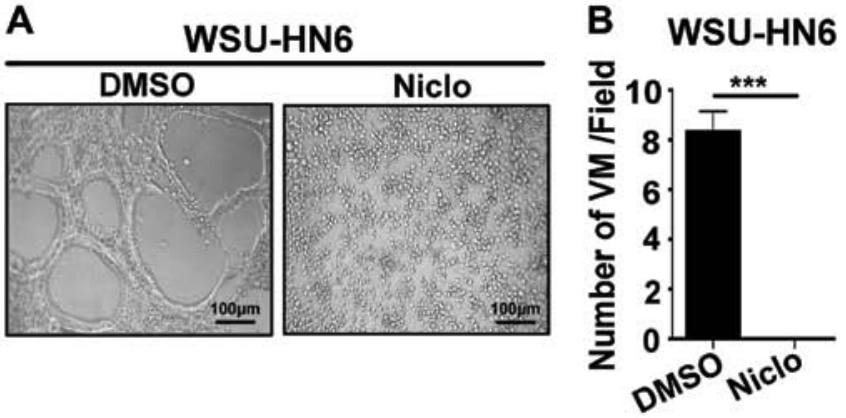

C
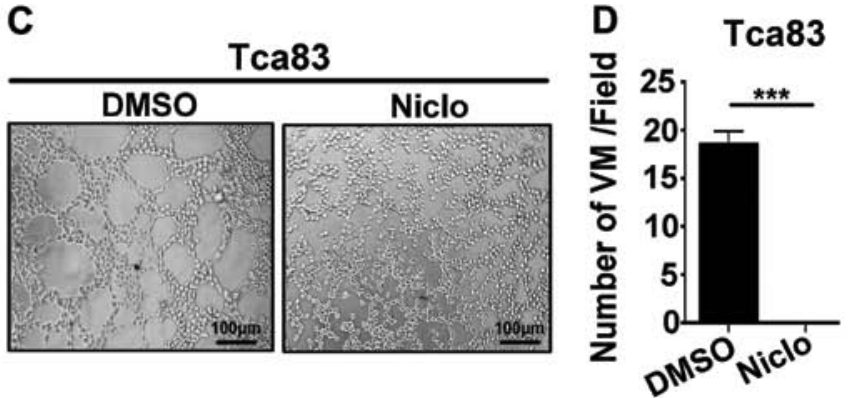

E

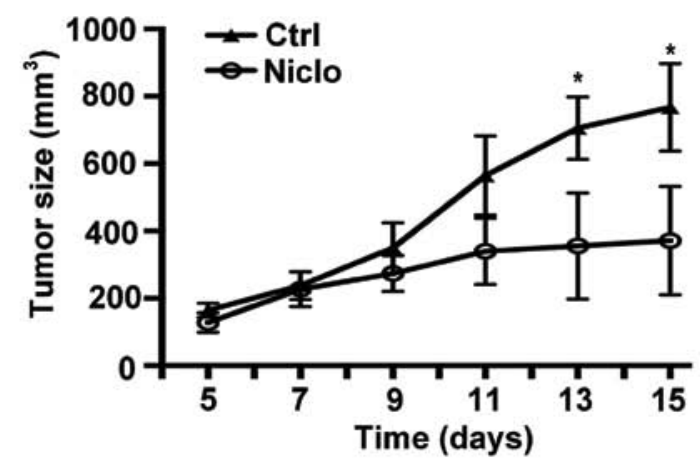

$\mathbf{F}$

F $\quad$ Ct

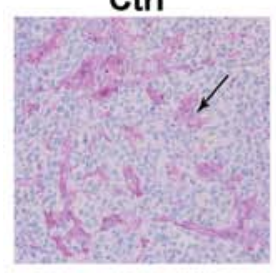

Niclo

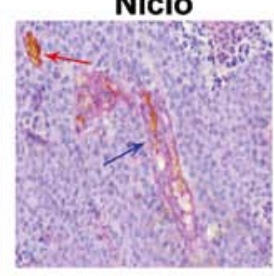

G

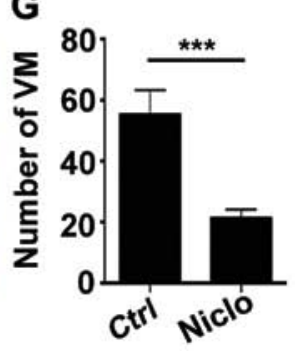

Figure 3. Niclosamide suppresses vasculogenic mimicry (VM) of cancer cells in vitro and in vivo. (A-D) Niclosamide significantly inhibits the capillary-like tube formation in WSU-HN6 and Tca83 cell lines. (E) Niclosamide significantly inhibits the tumor size in nude mice. Nude mice bearing WSU-HN6 cells were treated with vehicle control (Kolliphor ${ }^{\circledR}$ EL), niclosamide (20 mg/kg/day; i.p., injection). ( $F$ and G) Niclosamide decreases the number of VM compared with control group. Black arrow indicates VM; blue arrow indicates VM across blood vessel zone; and red arrow indicates blood vessel with only CD $34^{+}$stain, respectively $(\mathrm{x} 400) .{ }^{* * * *} \mathrm{P}<0.001$.

and Tca83 (Fig. 4B) cell lines. The western blot assay showed that niclosamide decreased the expression of VEGFA, MMP2, ROCK1 and p-Cdc42 at protein level in the two oral cancer cell lines (Fig. 4C-E).

miR-124 is involved in the anticancer effects of niclosamide through downregulation of $p$-STAT3 expression. To explore whether p-STAT3 and non-coding RNA is involved in the 
A

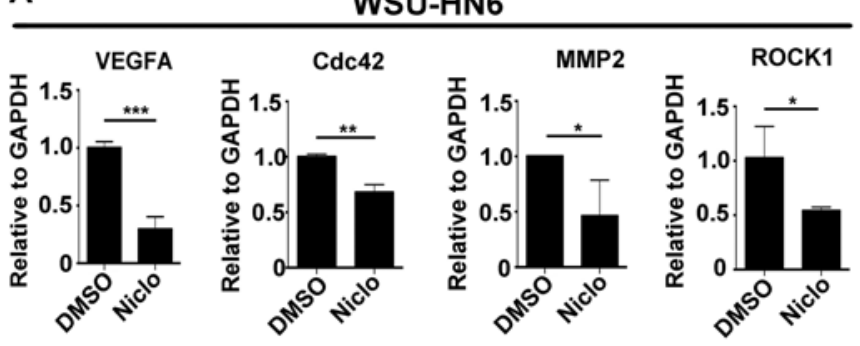

B

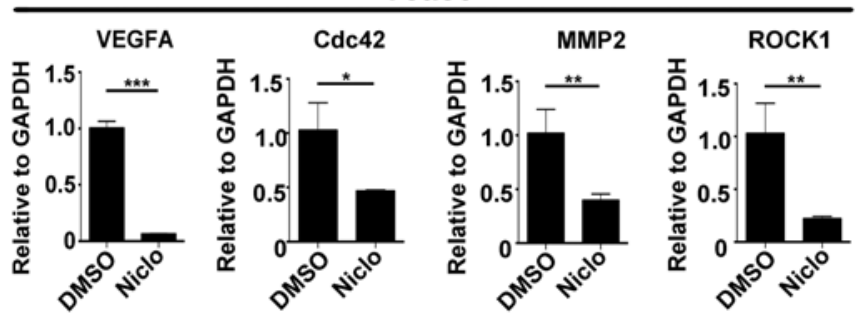

C
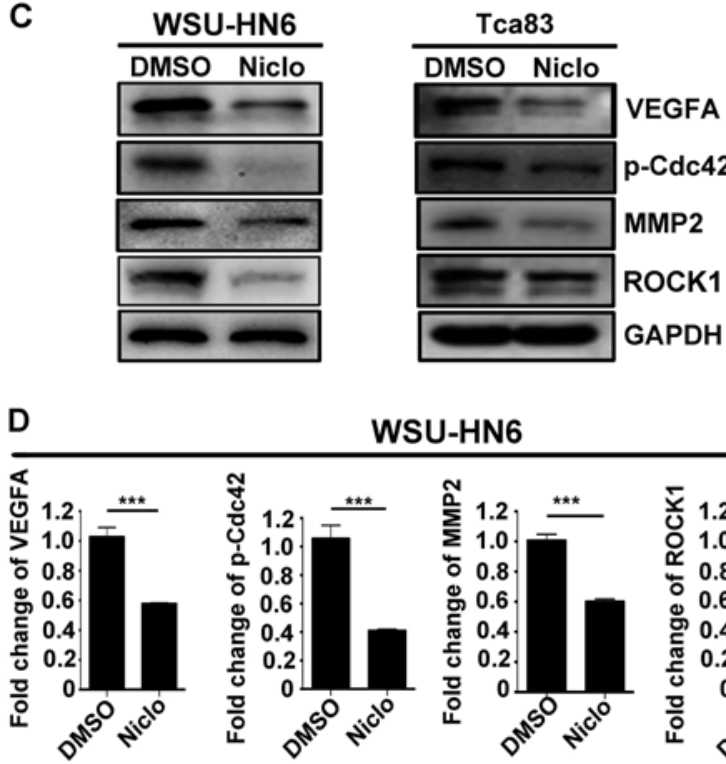

WSU-HN6

E
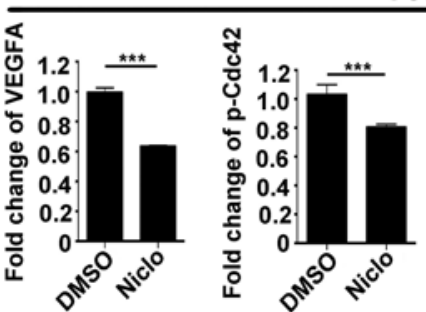

Tca83
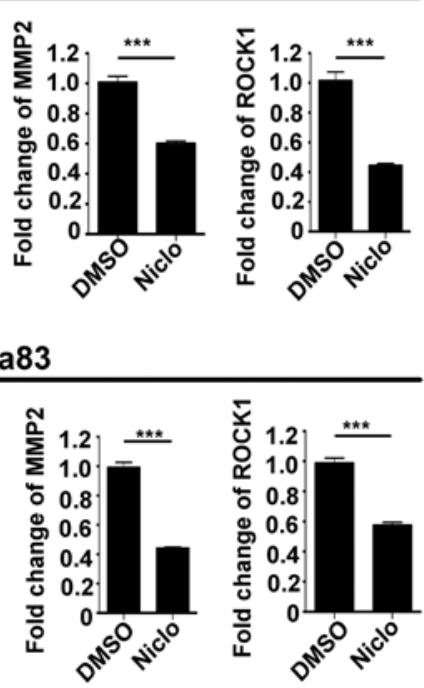

Figure 4. Niclosamide suppresses both vasculogenic mimicry (VM)-related genes and migration-related protein expression. Real-time PCR shows that VM-related genes VEGFA, MMP2, Cdc42 and ROCK1 are downregulated by niclosamide in WSU-HN6 (A) and Tca83 (B) at mRNA level. (C) Western blotting shows that niclosamide suppresses VEGFA, MMP2, p-Cdc42 and ROCK1 at protein level in the two oral cancer cell lines. (D and E) protein quantification by densitometry using Quantity One software (Bio-Rad), normalized to GAPDH ${ }^{*} \mathrm{P}<0.05,{ }^{* *} \mathrm{P}<0.01,{ }^{* * *} \mathrm{P}<0.001$.

anticancer effects of niclosamide, we performed western blotting and real-time PCR assays. The results showed that p-STAT3 was significantly downregulated at protein level

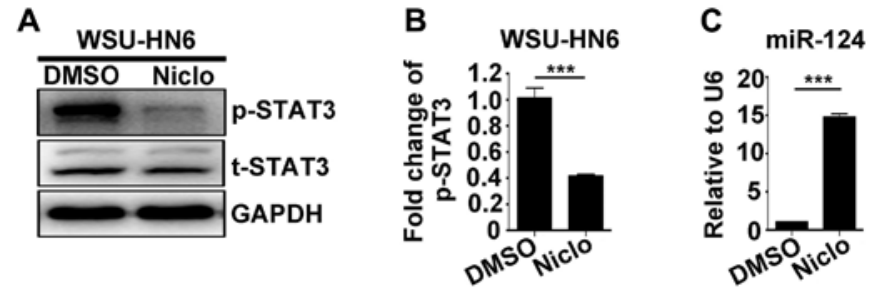

D

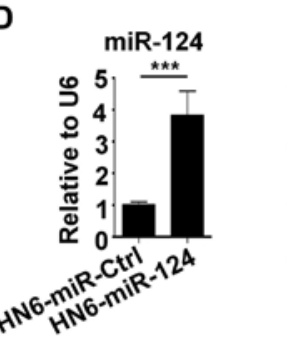

E $F$
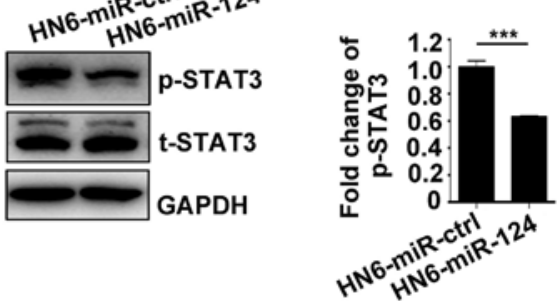

G

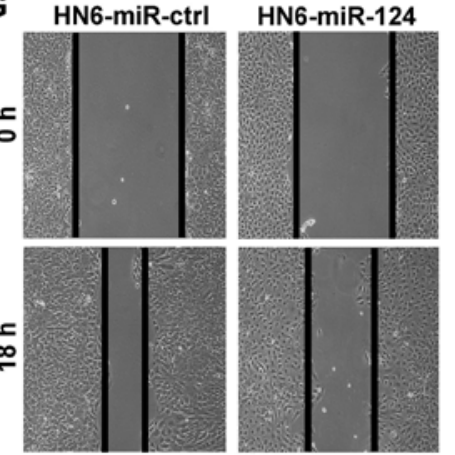

H

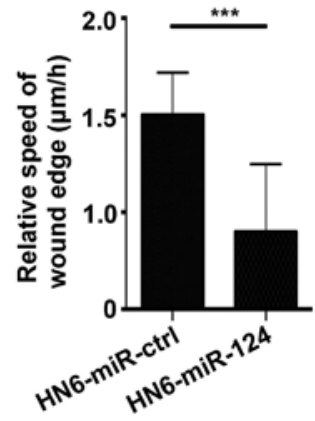

I
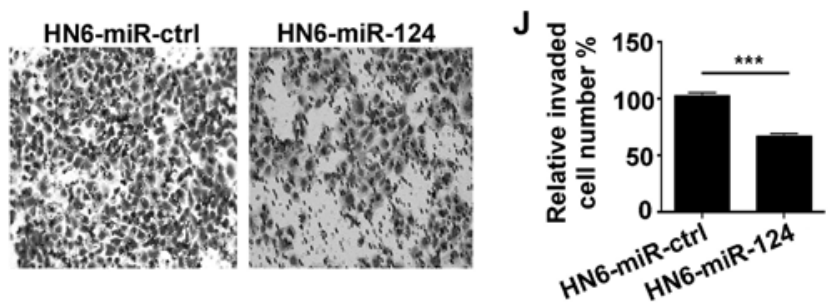

K

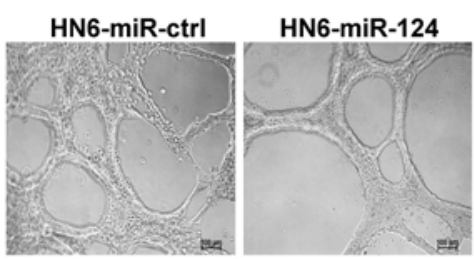

$\mathbf{L}$

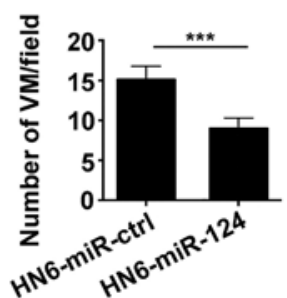

Figure 5. Niclosamide exerts anticancer effects by modulating vasculogenic mimicry (VM) through upregulation of miR-124 and downregulation of STAT3. (A and B) The expression of p-STAT3 is downregulated by $5 \mu \mathrm{M}$ niclosamide in WSU-HN6 cell line at protein level. (C) The expression of miR-124 is upregulated by $5 \mu \mathrm{M}$ niclosamide in WSU-HN6 cells at mRNA level. (D) miR-124 is upregulated in stable cell line HN6-miR-124. (E and F) The stable cell line HN6-miR-124 can downregulate the expression of p-STAT3. (G and H) HN6-miR-124 could decrease the ability of mobility of wound healing assay. (I and J) HN6-miR-124 could decrease the ability of invasiveness in Transwell assay. ( $\mathrm{K}$ and $\mathrm{L}$ ) Capillary-like tube formation assay shows that HN6-miR-124 decreases the number of capillary-like tube formation compared with control, ${ }^{* * *} \mathrm{P}<0.001$

(Fig. 5A and B); miR-124 was upregulated at mRNA level (Fig. $5 \mathrm{C}$ ) by $5 \mu \mathrm{M}$ niclosamide in WSU-HN6 cells. To further confirm the results, we generated stably overexpressing 
miR-124 cell line in WSU-HN6. The stable cell line HN6-miR-124 and empty vector control cell line were used to detect the expression of miR-124. Real-time PCR clearly showed that miR-124 was highly expressed in HN6-miR-124 (Fig. 5D), which suggested that HN6-miR-124 cell line was successfully established. In addition, p-STAT3 was downregulated in HN6-miR-124 compared with HN6-miR-ctrl cells (Fig. 5E and F). Furthermore, to investigate whether HN6-miR-124 affects the mobility, invasiveness and VM formation of oral cancer cells, we carried out wound healing, Transwell and capillary-like tube formation assays. The results show that HN6-miR-124 could significant reduce the migration (Fig. 5G and $\mathrm{H}$ ), invasion (Fig. 5I and $\mathrm{J}$ ) and the number of VM formation (Fig. 5K and L) compared with the HN6-miRctrl group.

\section{Discussion}

Bringing a new drug to market would take an average of 15 years and US $\$ 800$ million according to an analysis (18). In 2007, an article, entitled 'New uses for old drugs' by Chong and Sullivan (19) was published on 17 existing drugs that are in various stages of clinical and animal testing for new uses. Because of the known pharmacokinetics and safety profiles, it can bypass almost $40 \%$ of the overall cost of bringing a drug to market. Finding new uses for existing drugs is a proven low manufacturing costs and high stability, it is very attractive to be researched. In this study, we found a new anticancer mechanism of niclosamide through inhibition of vasculogenic mimicry (VM) formation.

VM is a tumor microcirculation pattern that usually exists in highly aggressive cancers. For example, the ability of highly invasive instead of poorly invasive, melanoma cells to generate patterned vascular channels in vitro (5). This helps to understand the strong association between the presence of vascular channel and poor cancer patient prognosis. Multiple molecular mechanisms, especially MMPs and VEGFA, have been reported to participate in VM formation which are regarded as significant factors in tumor migration and invasion. VEGFA belongs to an angiogenic growth factor family, and can be secreted by almost all tumor cells, and be associated with tumor angiogenesis. Expression of MMPs could be upregulated by VEGFA, contributing to matrix plasticity and VM formation (20). Furthermore, hypoxia is the most common phenomena. Hypoxia stimulates tumor cells secreting HIF-1 $\alpha$, which in turn activates VEGFA and contributes to VM formation (21). Traditional anti-angiogenic drugs targeted to endothelium reduce the density of blood vessel, as well as energy and oxygen supply, which can aggravate hypoxia of tumor cells. As tumors grow, the increasing nutrient and oxygen deficiency as a compensatory stimulus will contribute to VM formation and indirectly promote aggressiveness and therapeutic resistances of cancers. This can well explain why the common anti-angiogenic drugs have limited effect on VM.

To investigate the underlying mechanism that niclosamide could inhibit VM in vitro and in vivo, we explored the VM-related gene expression at mRNA and protein levels. We verified that while niclosamide inhibits VM, the expression of VEGFA, MMP2, ROCK1 and Cdc42 genes are downregu- lated (Fig. 4A). VEGFA and MMP2 play important roles in VM formation. ROCK and Cdc42 are cytoskeleton genes, which regulate the cell morphology, as well as epithelialmesenchymal transition (EMT), migration and invasion of cancer cells $(22,23)$. Research shows that ROCK is a regulator in VM in hepatocellular carcinoma cells (24) and osteosarcoma cells (25). Cdc42 is involved in VM, which can be downregulated by BNIP3 in melanoma cells (26). Thus, we can conclude that niclosamide could downregulate VM-related genes to inhibit VM formation. More importantly, VEGFA (27), MMP2 (28) and Cdc42 (29) can be regulated by STAT3.

STAT3 activation is frequently observed in advanced tumors and significantly involved in the process of proliferation, differentiation, apoptosis, migration and invasion of cancer cells (30), accumulating research shows that STAT3 can play an important role in angiogenesis both in physiological and pathological situations $(31,32)$. Furthermore, STAT3 activation plays a positive role in VM formation of gastric adenocarcinoma (33). Curcumin could suppress VM of laryngeal squamous cell carcinoma (34) and hepatocellular carcinoma (35) in vitro through the inhibition of STAT3 signaling pathway. Moreover, our results show that niclosamide could downregulate the expression of p-STAT3 at protein level. Based on the above data and evidence, we deduce that STAT3 may play a central role in the anticancer effects of niclosamide.

miRNAs are small noncoding RNAs that play important roles in many cellular processes (36). Up to this point, more than 30,424 human miRNAs have been identified and miRNAs usually function as potential oncogenes or oncosuppressor genes through imperfectly complementary to the 3' UTR of target mRNA $(37,38)$. In this study, we focused on miR-124 because it has been reported to be downregulated and affect metastasis in several types of cancer including oral cancer (39), suggesting that miR-124 has the potential as therapeutic target for oral cancer. However, there is no report on the effect of niclosamide on miR-124. In a recent study, our results show that niclosamide increases the expression of miR-124. Furthermore, reports suggested that there is interplay between miR-124 and STAT3 signaling pathway in multiple human cancer cells including endometrial carcinoma (40), hepatocellular carcinoma (41) and colorectal cancer (42). Therefore, we deduce that STAT3 may play an important role in the anticancer effects of niclosamide. To clarify the function of STAT3 and miR-124 in oral cancer, we detected the expression of p-STAT3 and the ability of VM in stable cell line HN6-miR-124. As our results show, such as niclosamide, HN6-miR-124 can decrease the expression of p-STAT3. Also, HN6-miR-124 has lower mobility, invasiveness and capillarylike tube formation ability compared with control. Based on the above data, we deduce that both miR-124 and STAT3 play key roles in the anticancer effects of niclosamide. Niclosamide exerts its anticancer effects partly through miR-124/STAT3/ $\mathrm{VM}$ axis.

In conclusion, our study, for the first time shows that niclosamide exerts its anticancer effects partly through modulating VM via upregulation of miR-124 and downregulation of STAT3. These findings support the possibility of using niclosamide as a target for anti-VM therapy in OSCC. 


\section{Acknowledgements}

This work was supported by the Research Fund for Capital Medical Development (2011-0425-02), the Research Grants from Nature Foundation of Heilongjiang Province (no. QC2014C107), Tianjin Natural Science Foundation (14JCQNJC12500), the National Nature Science Foundation of China (grant no. 81470707, 81300901 and 81772873) and Beijing Natural Science Foundation (7172240).

\section{References}

1. Jemal A, Ward EM, Johnson CJ, Cronin KA, Ma J, Ryerson B Mariotto A, Lake AJ, Wilson R, Sherman RL, et al: Annual Report to the Nation on the Status of Cancer, 1975-2014, Featuring Survival. J Natl Cancer Inst 109: djx030, 2017.

2. Zini A, Czerninski R and Sgan-Cohen HD: Oral cancer over four decades: Epidemiology, trends, histology, and survival by anatomical sites. J Oral Pathol Med 39: 299-305, 2010.

3. Yadav P: Recent advances in head and neck cancer reconstruction. Indian J Plast Surg 47: 185-190, 2014.

4. Hanahan D and Weinberg RA: Hallmarks of cancer: The next generation. Cell 144: 646-674, 2011.

5. Maniotis AJ, Folberg R, Hess A, Seftor EA, Gardner LM, Pe'er J, Trent JM, Meltzer PS and Hendrix MJ: Vascular channel formation by human melanoma cells in vivo and in vitro: Vasculogenic mimicry. Am J Pathol 155: 739-752, 1999.

6. Zhang S, Zhang D and Sun B: Vasculogenic mimicry: Current status and future prospects. Cancer Lett 254: 157-164, 2007.

7. van der Schaft DW, Seftor RE, Seftor EA, Hess AR, Gruman LM, Kirschmann DA, Yokoyama Y, Griffioen AW and Hendrix MJ: Effects of angiogenesis inhibitors on vascular network formation by human endothelial and melanoma cells. J Natl Cancer Inst 96 1473-1477, 2004

8. Xiang D, Yuan Y, Chen L, Liu X, Belani C and Cheng $\mathrm{H}$ : Niclosamide, an anti-helminthic molecule, downregulates the retroviral oncoprotein Tax and pro-survival Bcl-2 proteins in HTLV-1-transformed T lymphocytes. Biochem Biophys Res Commun 464: 221-228, 2015.

9. Chen W, Mook RA Jr, Premont RT and Wang J: Niclosamide: Beyond an antihelminthic drug. Cell Signal Apr 4, 2017 (Epub ahead of print).

10. Li Y, Li PK, Roberts MJ, Arend RC, Samant RS and Buchsbaum DJ: Multi-targeted therapy of cancer by niclosamide: A new application for an old drug. Cancer Lett 349: 8-14, 2014.

11. Djebali S, Davis CA, Merkel A, Dobin A, Lassmann T, Mortazavi A, Tanzer A, Lagarde J, Lin W, Schlesinger F, et al: Landscape of transcription in human cells. Nature 489: 101-108, 2012.

12. Martens-Uzunova ES, Böttcher R, Croce CM, Jenster G, Visakorpi T and Calin GA: Long noncoding RNA in prostate, bladder, and kidney cancer. Eur Urol 65: 1140-1151, 2014.

13. Li M, Marin-Muller C, Bharadwaj U, Chow KH, Yao Q and Chen C: MicroRNAs: Control and loss of control in human physiology and disease. World J Surg 33: 667-684, 2009.

14. Zhao Y, Ling Z, Hao Y, Pang X, Han X, Califano JA, Shan L and $\mathrm{Gu}$ X: MiR-124 acts as a tumor suppressor by inhibiting the expression of sphingosine kinase 1 and its downstream signaling in head and neck squamous cell carcinoma. Oncotarget 8 : 25005-25020, 2017

15. Chen SM, Chou WC, Hu LY, Hsiung CN, Chu HW, Huang YL, Hsu HM, Yu JC and Shen CY: The effect of MicroRNA-124 overexpression on anti-tumor drug sensitivity. PLoS One 10: e0128472, 2015 .

16. Wan HY, Li QQ, Zhang Y, Tian W, Li YN, Liu M, Li X and Tang H: MiR-124 represses vasculogenic mimicry and cell motility by targeting amotL1 in cervical cancer cells. Cancer Lett 355: 148-158, 2014

17. Chiang HR, Schoenfeld LW, Ruby JG, Auyeung VC, Spies N Baek D, Johnston WK, Russ C, Luo S, Babiarz JE, et al Mammalian microRNAs: Experimental evaluation of novel and previously annotated genes. Genes Dev 24: 992-1009, 2010.

18. DiMasi JA, Hansen RW and Grabowski HG: The price of innovation: New estimates of drug development costs. J Health Econ 22: 151-185, 2003

19. Chong CR and Sullivan DJ Jr: New uses for old drugs. Nature 448: 645-646, 2007.
20. Wang JY, Sun T, Zhao XL, Zhang SW, Zhang DF, Gu Q, Wang XH, Zhao N, Qie S and Sun BC: Functional significance of VEGF-a in human ovarian carcinoma: Role in vasculogenic mimicry. Cancer Biol Ther 7: 758-766, 2008.

21. Jones MK, Szabó IL, Kawanaka H, Husain SS and Tarnawski AS: von Hippel Lindau tumor suppressor and HIF-1alpha: New targets of NSAIDs inhibition of hypoxia-induced angiogenesis. FASEB J 16: 264-266, 2002.

22. Feltrin D and Pertz O: Assessment of Rho GTPase signaling during neurite outgrowth. Methods Mol Biol 827: 181-194, 2012.

23. Kwon J, Kim NH and Choi I: ROCK activity regulates functional tight junction assembly during blastocyst formation in porcine parthenogenetic embryos. PeerJ 4: e1914, 2016.

24. Zhang JG, Li XY, Wang YZ, Zhang QD, Gu SY, Wu X, Zhu GH, Li Q and Liu GL: ROCK is involved in vasculogenic mimicry formation in hepatocellular carcinoma cell line. PLoS One 9: e107661, 2014

25. Xia Y, Cai X, Fan J, Zhang L, Li Z, Ren J, Wu G and Zhu F: RhoA/ ROCK pathway inhibition by fasudil suppresses the vasculogenic mimicry of U2OS osteosarcoma cells in vitro. Anticancer Drugs 28: 514-521, 2017.

26. Maes H, Van Eygen S, Krysko DV, Vandenabeele P, Nys K, Rillaerts K, Garg AD, Verfaillie T and Agostinis P: BNIP3 supports melanoma cell migration and vasculogenic mimicry by orchestrating the actin cytoskeleton. Cell Death Dis 5: e1127, 2014.

27. Chen $Z$ and Han ZC: STAT3: A critical transcription activator in angiogenesis. Med Res Rev 28: 185-200, 2008.

28. Zhu W, Sun W, Zhang JT, Liu ZY, Li XP and Fan YZ: Norcantharidin enhances TIMP-2 anti-vasculogenic mimicry activity for human gallbladder cancers through downregulating MMP-2 and MT1-MMP. Int J Oncol 46: 627-640, 2015.

29. Wu F, Chen Y, Li Y, Ju J, Wang Z and Yan D: RNA-interferencemediated Cdc42 silencing down-regulates phosphorylation of STAT3 and suppresses growth in human bladder-cancer cells. Biotechnol Appl Biochem 49: 121-128, 2008.

30. Yu H and Jove R: The STATs of cancer - new molecular targets come of age. Nat Rev Cancer 4: 97-105, 2004.

31. Valdembri D, Serini G, Vacca A, Ribatti D and Bussolino F: In vivo activation of JAK2/STAT-3 pathway during angiogenesis induced by GM-CSF. FASEB J 16: 225-227, 2002.

32. Osugi T, Oshima Y, Fujio Y, Funamoto M, Yamashita A, Negoro S, Kunisada K, Izumi M, Nakaoka Y, Hirota H, et al: Cardiac-specific activation of signal transducer and activator of transcription 3 promotes vascular formation in the heart. J Biol Chem 277: 6676-6681, 2002.

33. Song YY, Sun LD, Liu ML, Liu ZL, Chen F, Zhang YZ, Zheng Y and Zhang JP: STAT3, p-STAT3 and HIF-1 $\alpha$ are associated with vasculogenic mimicry and impact on survival in gastric adenocarcinoma. Oncol Lett 8: 431-437, 2014.

34. Hu A, Huang JJ, Jin XJ, Li JP, Tang YJ, Huang XF, Cui HJ, $\mathrm{Xu} \mathrm{WH}$ and Sun GB: Curcumin suppresses invasiveness and vasculogenic mimicry of squamous cell carcinoma of the larynx through the inhibition of JAK-2/STAT-3 signaling pathway. Am J Cancer Res 5: 278-288, 2014

35. Chiablaem K, Lirdprapamongkol K, Keeratichamroen S, Surarit R and Svasti J: Curcumin suppresses vasculogenic mimicry capacity of hepatocellular carcinoma cells through STAT3 and PI3K/AKT inhibition. Anticancer Res 34: 1857-1864, 2014.

36. Qureshi IA and Mehler MF: Regulation of non-coding RNA networks in the nervous system - what's the REST of the story? Neurosci Lett 466: 73-80, 2009.

37. Carroll AP, Tooney PA and Cairns MJ: Context-specific microRNA function in developmental complexity. J Mol Cell Biol 5: 73-84, 2013.

38. Kozomara A and Griffiths-Jones S: miRBase: Annotating high confidence microRNAs using deep sequencing data. Nucleic Acids Res 42 (Database issue): D68-73, 2014.

39. Jin L, Miao J, Liu Y, Li X, Jie Y, Niu Q and Han X: Icaritin induces mitochondrial apoptosis by up-regulating miR-124 in human oral squamous cell carcinoma cells. Biomed Pharmacother 85: 287-295, 2017.

40. Li Y, Zhang Z, Liu X, Huang T, He W, Shen Y, Liu X, Hong K and Cao Q: miR-124 functions as a tumor suppressor in the endometrial carcinoma cell line HEC-1B partly by suppressing STAT3. Mol Cell Biochem 388: 219-231, 2014.

41. Lu Y, Yue X, Cui Y, Zhang J and Wang K: MicroRNA-124 suppresses growth of human hepatocellular carcinoma by targeting STAT3. Biochem Biophys Res Commun 441: 873-879, 2013.

42. Zhang J, Lu Y, Yue X, Li H, Luo X, Wang Y, Wang K and Wan J: MiR-124 suppresses growth of human colorectal cancer by inhibiting STAT3. PLoS One 8: e70300, 2013. 